NYU-TH-08/10/62

\title{
Effective Lagrangian and Quantum Screening in Charged Condensate
}

\author{
Gregory Gabadadze and Rachel A. Rosen \\ Center for Cosmology and Particle Physics, Department of Physics, \\ New York University, New York, NY 10003, USA
}

\begin{abstract}
A condensate of charged scalars in a neutralizing background of fermions (e.g., condensed helium-4 nuclei in an electron background in white dwarf cores) is investigated further. We discuss an effective Lagrangian approach to this system and show that the strong screening of an electric charge found previously in arXiv:0806.3692 in a mean-field approximation, is a consequence of a cancellation due to a phonon. The resulting propagators contain terms that strongly modify their infrared behavior. Furthermore, we evaluate a one-loop fermion quantum correction to the screened potential, and find that it is also suppressed by the phonon subtraction. Therefore, charged impurities (e.g., hydrogen or helium-3 nuclei) will be screened efficiently by the condensate.
\end{abstract}




\section{Introduction and Summary}

Consider a system of negatively charged fermions (e.g., electrons) of mass $m_{f}$ and of number-density $J_{0}$, and positively charged scalars (e.g., helium-4 nuclei) of mass $m_{H}$, with the net charge of the system being zero. At densities $J_{0} \simeq(0.5-5 \mathrm{MeV})^{3}$ the average inter-fermion separation, $d \sim J_{0}^{-1 / 3} \sim(400-40 \mathrm{fm})$, is much smaller than the atomic scale $\sim 10^{5} \mathrm{fm}$, while being much greater than the nuclear scale $\sim 1 \mathrm{fm}$. Therefore, neither atomic nor nuclear effects would matter at such densities. The fermions would form a degenerate gas with the Fermi energy exceeding interfermion repulsion energy, which can be ignored. These are the conditions thought to be present in cores of helium white dwarfs.

As a helium dwarf star cooled from $\sim 10^{6}-10^{7} \mathrm{~K}$, down to lower temperatures, the helium-4 nuclei could have formed a crystalline structure. However, the zeropoint oscillations of helium-4 ions at these densities would "melt" such a crystal, and it was proposed $[1,2]$, that the helium-4 ions could instead condense into a macroscopic state of large occupation number - the charged condensate - specific properties of which were discussed in detail in [2].

In particular, a static potential between probe charges placed in the condensate were calculated in [2] in the mean-field approximation, and were shown to be suppressed by the exponential factor, $\sim \exp (-M r) \cos (M r) / r$, where the scale $M$ is defined in terms of the electric charge $e$, mass $m_{H}$, and number-density $J_{0}$ :

$$
M \equiv\left(2 e^{2} m_{H} J_{0}\right)^{1 / 4}>J_{0}^{1 / 3} .
$$

Although this strong screening may well be a reason why the condensation of charged bosons takes place in the first place, the following issues emerge in this regard:

(i) It may seem that the exponent $\exp (-M r)$ is due to a state of mass $M$. The distance scale $1 / M$ is shorter than the average inter-particle separation - an effective short-distance cutoff of the low-energy theory. Then, a state of mass $M$, if existed, would have been beyond the scope of the low-energy field theory description, and the above-cited potential would have been unreliable.

(ii) The momentum-space static potential, the Fourier transform of which is proportional to $\sim \exp (-M r) \cos (M r) / r$, reads as follows (see Section 3)

$$
G(\omega=0, \mathbf{k}) \simeq\left(\mathbf{k}^{2}+m_{0}^{2}+\frac{4 M^{4}}{\mathbf{k}^{2}}\right)^{-1}
$$

where, $m_{0}^{2}$ stands for a certain quantity such that $\left|m_{0}^{2}\right| \ll M^{2}$ (see Ref. [2] and Sections 3,4 below). The term $M^{4} / k^{2}$ gives rise to a significant modification of the Green's function in the infrared. The interpretation of such an infrared-sensitive term in the denominator of a propagator calls for an explanation.

(iii) In the $m_{H} \rightarrow \infty$ limit one would expect the heavy scalars (helium-4 nuclei) to decouple. It is not exactly clear from (2) how such a decoupling takes place, and what is its interpretation. 
The above three issues, can be clarified, as we will summarize below, and show in this paper. The points (i) and (ii) get resolved by observing that the Green's function (2) can formally be decomposed as follows:

$$
G(\mathbf{k}, \omega=0) \simeq \frac{1}{\mathbf{k}^{2}+m_{0}^{2}}-\frac{1}{\mathbf{k}^{2}+m_{0}^{2}+\mathbf{k}^{2}\left(\mathbf{k}^{2}+m_{0}^{2}\right)^{2} / 4 M^{4}} .
$$

The first term on the r.h.s. can be thought of as an instantaneous screened Coulomb potential, while the second one can be interpreted as a potential due to a phonon. The phonon in this case is a collective excitation of motion of charged scalars within the fermion background. As was shown in [2], the phonon is a light excitation that belongs to the spectrum of a low-energy effective field theory. It's just the cancellation due to this light mode that gives rise to the exponential $\exp (-M r)$, and not a hypothetical state of mass $M$.

Before we turn to the point (iii) let us make two important comments. First, we should note that the second term in (3) has three poles. The residue of the pole at $\mathbf{k}^{2}+m_{0}^{2}=0$ cancels exactly with that from the first term of (3). The remaining two poles, describe both the heavy state of mass $\sim m_{H}$, and a light state, which actually is the phonon [2]. For simplicity of the discussions, in this work we'll be using a somewhat arbitrary language by calling the whole second term in (3) the phonon contribution.

Second, we note that the form of (3) may be puzzling itself: the negative sign in front of the second term on the r.h.s. may seem to be suggesting that the phonon is a ghost. This indeed would have been a ghost contribution if we were to obtain such a term in a spectrum of a Lorentz-invariant theory. Here, the background solution fixes the Lorentz frame, and the spectrum of small perturbations is not Lorentzinvariant [1]. Because of this, the negative sign in front of the phonon Green's functions is not a signature of a ghost. In particular, as we'll show in Section 3, the Hamiltonian for the fluctuations that give rise to (3) is positive semi-definite! Moreover, the measure of Lorentz-breaking is the scale $M$. In the $M \rightarrow 0$ limit, the phonon term in (3) vanishes, as one would expect.

Let us now turn to the point (iii). In the limit $m_{H} \rightarrow \infty$, which implies $M \rightarrow \infty$, the phonon effects should go away. This is reflected in (3) as vanishing of the whole potential, which is just a consequence of using the static approximation, and can be understood in the following way: The phonon mixes with the timelike component of the gauge field, and due to this acquires an instantaneous part. Then, the instantaneous parts in (3) cancel between the gauge and photon contributions. However, the dynamical part of the phonon is also reducing to zero, because the group velocity of the phonon vanishes in the $m_{H} \rightarrow \infty$ limit, as we will show in Section 3.

Having the above properties clarified, in this work we will study further the static charge screening in charged condensate. The physical question is that of static interactions between charged impurities placed into the condensate. As we mentioned above, the scalar condensate gives rise to the suppression of the static 
potential by a factor $\exp (-M r)$. Inclusion of the fermion fluctuations via the meanfield Thomas-Fermi method, does not change the above result significantly [2].

In Section 4 we go beyond the mean-field approximation and include a one-loop quantum correction due to the polarization diagram. This diagram is suppressed by an additional power of the electromagnetic coupling constant $\alpha_{\mathrm{em}}=e^{2} / 4 \pi$, and one would expect the quantum correction to be insignificant.

However, this is not the case for the following subtle reason. The one-loop correction introduces branch cuts in the Green's function (3), which give rise to additional contributions to the static potential in the position space. These additional terms have oscillatory nature with a power-like decaying envelope. Even though they are formally suppressed by $\mathcal{O}\left(\alpha_{\mathrm{em}}^{2}\right)$, to a good approximation they end up being $\mathcal{O}(1)$, and can dominate over the exponentially suppressed term at sufficiently large distances.

Such an oscillatory potential is know in non-relativistic fermion systems as the Friedel potential (for original references see [3]), which was generalized to relativistic fermions by Sivak [4] and by Kapusta and Toimela [5]. Here we perform analogous calculations for the charged condensate and find that the phonon subtraction suppresses further the Friedel-like potential by the factor $J_{0}^{8 / 3} / M^{8} \sim 10^{-23}$. In spite of this, for reasonable separations between the test particles, the Friedel-like potential dominates over the exponential one.

Therefore, charged impurities, such as hydrogen or helium-3 nuclei, once placed within the charged condensate, will interact very weakly with the ambient condensate and with each other. The impurities are screened very efficiently by the condensate!

Let us make a few comments on the literature. Condensation of non-relativistic charged scalars has a long history, the original works being those by Schafroth [6], in the context of superconductivity, and by Foldy [7], in a more general setup. An almost-ideal Bose gas approximations was assumed in those studies. For this assumption to be true, densities had to be taken high-enough to make the average inter-particle separation shorter than the boson Bohr radius [7]. In terms of our parameters this would be the case if $J_{0}^{1 / 3} \gtrsim \alpha_{\mathrm{em}} m_{H}$.

However, for the helium-electron system the above condition would translate into super-high densities, at which nuclear interactions become significant. Instead, in this work we're studying charged condensation in the opposite regime, $J_{0}^{1 / 3} \ll$ $\alpha_{\mathrm{em}} m_{H}$, where the nuclear forces play no role. Moreover, our mechanism of screening the electric charge is different, as it is based on cancellation by the phonon.

A possibility of having a changed condensate in helium white dwarfs was previously discussed in Ref. $[8]^{1}$, where the condensation was studied using an approximate variational quantum-mechanical calculation in conjunction with numerical

\footnotetext{
${ }^{1}$ We thank Andrei Gruzinov for bringing this paper to our attention. Regretfully, this happened after our work on charge condensation in white dwarfs [2] was already published. We use this opportunity here to emphasize differences between our approach and that of [8].
} 
insights in a strongly-coupled regime of electromagnetic interactions. A degree of reliability of such a scheme is hard to assess ${ }^{2}$. Finally, in the context of a relativistic field theory condensation of scalars was discussed in, e.g., Refs. [9, 10, 11], some of the results of which we cite below.

A. Dolgov, A. Lepidi, and G. Piccinelli [12] (appeared on archive the same day with this paper) calculated a one-loop correction to the photon self-energy in the background of charged condensate at nonzero temperature. These author find similar infrared modifications of the static potential as we did. Moreover, their results also include nonzero temperature effects, and in that part, are more general than ours. On the other hand, our emphasis is on understanding of these results in terms of the phonon and effective Lagrangian language.

\section{Effective Lagrangian and its Solution}

In this Section we discuss an effective Lagrangian description of charged condensation. We will treat the bosonic and fermionic degrees of freedom separately. This has two reasons: (a) There is a significant disparity of physical scales in these two sectors; (b) Due to the Fermi surface, the dynamics for fermionic fluctuations should be though of in terms of near-the-Fermi-surface degrees of freedom. For instance, when the energy of these excitations scales to zero, their momenta scale to the Fermi surface [13]. To be able to describe the dynamics of the near-the-Fermi-surface fluctuations, including their one-loop quantum polarization effects, we will adopt for them a microscopic Lagrangian with a relativistic chemical potential

$$
\bar{\psi}\left(i \gamma^{\mu} D_{\mu}-m_{f}\right) \psi+\mu_{f} \psi^{+} \psi
$$

where the covariant derivative for fermions (electrons) is defined as $D_{\mu}=\partial_{\mu}+i e A_{\mu}$, and $\mu_{f}$ denotes the fermion chemical potential, that equals to the Fermi energy at zero temperature $\mu_{f}=\epsilon_{F}=\left[\left(3 \pi^{2} J_{0}\right)^{2 / 3}+m_{f}^{2}\right]^{1 / 2}$.

For the bosonic sector, on the other hand, we will employ the effective orderparameter description. In particular we will look for classical solutions of the equations of motion of the effective order-parameter Lagrangian.

How could a classical solution describe the condensation which is an inherently quantum phenomenon? Denote the particle creation and annihilation operators by $a_{0}^{+}$and $a_{0}$ respectively; then, the quantum-mechanical noncomutativity of these operators, $a_{0}^{+} a_{0}-a_{0} a_{0}^{+} \sim \hbar$, becomes an insignificant effect of order $\mathcal{O}(\hbar / N)$, when the number of particles in the condensate state, $\left\langle a_{0}^{+} a_{0}\right\rangle \sim N$, is large enough, $N \gg 1$. Thus, the classical description of the coherent state with a large occupation number

\footnotetext{
${ }^{2}$ Furthermore, using the ordinary neutral Bose-Einstein (BE) condensation to describe the charged condensate, as it is done in a number of works in the literature, is unjustified. Properties of a neutral BE condensate differ significantly from those of the charged condensate considered here. For instance, specific heat at moderate temperatures in the former is due to a phonon gas, while in the latter it is due to the degenerate electrons (see, e.g., [2]).
} 
- the condensate - should be valid to a good accuracy [14]. On the other hand, collective excitations of the condensate itself should be quantized in a conventional manner.

The above arguments lead to the following decomposition of the order-parameter operator describing the condensate:

$$
\Phi=\Phi_{c l}+\delta \Phi
$$

where $\Phi_{c l}$ denotes just a classical solution of the corresponding equations of motion, and describes the condensate of many zero-momentum particles, while $\delta \Phi$ should describe their collective fluctuations.

In the non-relativistic approximation in $m_{H}$, the effective order-parameter Lagrangian that is consistent with the translational, rotational, Galilean and the global $U(1)$ (scalar number) symmetries, as well as with local gauge invariance, can be written as follows:

$$
\mathcal{L}_{e f f}=\mathcal{P}\left(\frac{i}{2}\left(\Phi^{*} D_{0} \Phi-\left(D_{0} \Phi\right)^{*} \Phi\right)-\frac{\left|D_{j} \Phi\right|^{2}}{2 m_{H}}\right)
$$

where $D_{0} \equiv\left(\partial_{0}-i 2 e A_{0}\right), D_{j} \equiv\left(\partial_{j}-i 2 e A_{j}\right)$, while $\mathcal{P}(x)$ stands for a general polynomial function of its argument. The coefficients of this polynomial are dimensionful numbers that are inversely proportional to powers of a short-distance cutoff of the effective field theory, $\mathcal{P}(x)=\sum_{n=0}^{\infty} c_{n}\left(x^{n} / \Lambda^{3 n}\right)$.

Such a Lagrangian was first proposed by Greiter, Wilczek and Witten (GWW) [15] in a context of superconductivity. The requirements leading to (6) are the following: (a) In the lowest order in fields it gives rise to the standard Schrödinger equation for the order parameter ${ }^{3}$; (b) It respects all the appropriate symmetries of the physical system at hand, and gives rise to an operator relation between a current of charge $q$ and the momentum density $J_{j}=\left(q / m_{H}\right) T_{0 j}$. (c) It gives an appropriate spectrum of Nambu-Goldstone bosons in the decoupling limit ${ }^{4}$.

The GWW effective Lagrangian can also describe charged condensation, as we're about to show. For this we use the representation of the order parameter in terms of its modulus and the phase, (which is well-defined everywhere since the modulus will have a nonzero $\mathrm{VEV})$ :

$$
\Phi=\Sigma \exp (i \Gamma)
$$

\footnotetext{
${ }^{3}$ In our nomenclature electrons are "particles" while helium- 4 nuclei are "antiparticles", this explains the choice of the plus sing in front of the first term in (6).

${ }^{4}$ One could add to the argument of the function $\mathcal{P}(x)$ in $(6)$ terms $\mu_{N R} \Phi^{*} \Phi, \lambda\left(\Phi^{*} \Phi\right)^{2}$, etc. The former could be absorbed into a gauge potential $A_{0}$ by a constant shift, but in any case, in the condensation phase, which is a point of the primary interest here, the non-relativistic chemical potential $\mu_{N R}$, should be zero (or equivalently, the relativistic chemical potential $\mu_{s}=\mu_{N R}+m_{H}$ should exactly equal to the particle mass $\mu_{s}=m_{H}$ ). Furthermore, the existence of the quartic term for our system would not play an important role as long as $\lambda \lesssim 1$ and $J_{0} \ll m_{H}^{3}$.
} 
Using this decomposition we rewrite the argument of the function $\mathcal{P}$ and the effective Lagrangian (6) in the following form:

$$
\mathcal{L}_{e f f}=\mathcal{P}\left(2 e \Sigma^{2} B_{0}-\frac{\left(\nabla_{j} \Sigma\right)^{2}+(2 e)^{2} \Sigma^{2} B_{j}^{2}}{2 m_{H}}\right),
$$

where we introduced a gauge-invariant field $B_{\mu} \equiv A_{\mu}-\partial_{\mu} \Gamma / 2 e$. Varying (8) w.r.t. $\Sigma$ and $A_{0}$, and restricting ourselves only to constant fields, we obtain the corresponding equations:

$$
\mathcal{P}^{\prime} \Sigma\left(4 e B_{0}-\frac{(2 e)^{2} B_{j}^{2}}{m_{H}}\right)=0, \quad 2 \mathcal{P}^{\prime} \Sigma^{2}=J_{0},
$$

where in the last equation we took into account that the gauge field also couples to the background fermion charge density $e J_{0}$ as follows: $-e A_{0} J_{0}$. Note, that for space-time constant fields the equation of motion obtained by variation of (8) w.r.t. $\Gamma$ is satisfied identically.

There exists a solution to these equations for which

$$
2 \Sigma^{2}=J_{0}, \quad B_{\mu}=0, \quad \mathcal{P}^{\prime}(0)=1
$$

Since on the solution the argument of (8) is zero, the condition $\mathcal{P}^{\prime}(0)=1$ is satisfied by any polynomial functions $\mathcal{P}(x)$ for which the first coefficient is normalized to one

$$
\mathcal{P}(x)=x+C_{2} x^{2}+\ldots
$$

The above solution describes a neutral system of negatively charged electrons of charge density $-e J_{0}$, and positively charged scalar (helium-4 nuclei) condensate of charge density $2 e \Phi^{+} \Phi=2 e \Sigma^{2}=e J_{0}$.

Let us now turn to the issue of fluctuations about the classical solution. In Ref. [15] the Lagrangian (6) was proposed for the description of low-energy theory of superconductivity, where a phonon has a linear dispersion relation $\omega \propto|\mathbf{k}|$, for small $|\mathbf{k}|$. Such a relation was obtained in [15] by fixing $\Sigma(x)$ to a constant, but allowing for fluctuations of the phase $\Gamma$.

The system considered here is different, however. In the limit of switched-off Coulomb interactions (decoupling limit) it should reduce to a collection of free particles of mass $m_{H}$. The latter undergoes Bose-Einstein condensation at low temperatures. The resulting condensate exhibits no super-conductivity/fluidity because of the lack of interactions between particles. Hence, the dispersion relation for the first possible excitation in this system should be $\omega \simeq \mathbf{k}^{2} / 2 m_{H}$.

Remarkably, this dispersion relation can be obtained from the GWW Lagrangian (6) by allowing for the $\Sigma(x)$ field to have small fluctuations. The latter mix with the fluctuations of the phase, and, as a result, gives rise to the dispersion relation $\omega \simeq \mathbf{k}^{2} / 2 m_{H}$, as we will show in the next Section. 
The above arguments suggest that taking a non-relativistic limit to decouple a dynamics of a heavy mode in the presence of a condensate, is a bit subtle, as is well known in solid state theory: For instance, even though the ions in a simple crystal lattice could be very heavy and non-relativistic, there still could exists a low-energy mode, a phonon, with a linear dispersion relation $\omega \sim|\mathbf{k}|$, associated with a collective motion of those heavy ions. The decoupling of heavy ions in the non-relativistic limit manifest itself as vanishing of the group velocity of the phonon in the infinite-ion-mass limit.

A similar effect for a many-body system of free bosons can be sketched as follows: Consider a single massive state with the dispersion $\left(\frac{\omega}{c}\right)^{2}=\mathbf{k}^{2}+m_{H}^{2} c^{2}$. To describe a many-body system of such particles one could replace $\omega$ by $\omega+\mu_{s} c$, where $\mu_{s}$ is the chemical potential, and put $\mu_{s}$ equals to $m_{H}$, in order to describe condensation. Then, the dispersion relation becomes $\left(\frac{\omega}{c}\right)^{2}=\mathbf{k}^{2}-2 \omega m_{H}$. From this, one gets a dispersion relation in the non-relativistic limit: $\omega=\mathbf{k}^{2} / 2 m_{H}$, with the group velocity $v_{\mathrm{gr}}=|\mathbf{k}| / m_{H}$ being a $\mathcal{O}\left(1 / m_{H}\right)$ effect, consistently with ones expectation and the heavy mass decoupling.

For convenience, in what follows we will start our discussions with a relativistic Lorentz-invariant Lagrangian that retains the heavy scalar mode. Normally, this mode would not be included in the effective Lagrangian, and we should be careful to separate heavy and light modes in our calculations, and use only the light ones for the discussion of physical effects. Thus, the full Lagrangian containing the relativistic order-parameter $\phi$, gauge boson, and fermions read as follows:

$$
\mathcal{L}=-\frac{1}{4} F_{\mu \nu}^{2}+\left|\tilde{D}_{\mu} \phi\right|^{2}-m_{H}^{2} \phi^{*} \phi+\bar{\psi}\left(i \gamma^{\mu} D_{\mu}-m_{f}\right) \psi+\mu_{f} \psi^{+} \psi
$$

A classical nonzero vacuum expectation value of the field $\phi$ can serve as an order parameter for the condensation of the helium-4 nuclei, thus describing a state with a large occupation number. Fluctuations of the order parameter are expected to describe the collective modes of the condensate ${ }^{5}$.

The covariant derivative for the scalar reads $\tilde{D}_{\mu} \equiv \partial_{\mu}-2 i e \tilde{A}_{\mu} \equiv \partial_{\mu}-i\left(2 e A_{\mu}+\right.$ $\left.\mu_{s} \delta_{\mu 0}\right)$, where $\mu_{s}$ is the relativistic chemical potential for the scalars. The Lagrangian (12) is invariant under global $U_{s}(1)$ transformations, responsible for the conservation of the number of scalars. Another global $U_{f}(1)$ guarantees the fermion number conservation. One linear combination of these two symmetries is gauged, and the corresponding conserved current is coupled to the photon field ${ }^{6}$.

As before, we introduce the representation for the scalar field, $\phi=\frac{1}{\sqrt{2}} \sigma e^{i \alpha}$, and work in the unitary gauge where the phase of the scalar is set to zero, $\alpha=0$. In

\footnotetext{
${ }^{5}$ The Lagrangian (12) is more restrictive than it's needed - the effective Lagrangian (6) allows for a more general velocities of propagation than the Lorentz-invariant one. However, this degeneracy of (12) will be lifted by the fermion fluctuations, see Sections 3 and 4 .

${ }^{6}$ We could add the quartic scalar self-interaction term to (12), but this won't change our results significantly, as long as the quartic coupling is not strong, and $m_{H} \gg J_{0}^{1 / 3}$.
} 
this gauge, the Lagrangian density reads:

$$
\mathcal{L}=-\frac{1}{4} F_{\mu \nu}^{2}+\frac{1}{2}\left(\partial_{\mu} \sigma\right)^{2}+\frac{(2 e)^{2}}{2} \tilde{A}_{\mu}^{2} \sigma^{2}-\frac{1}{2} m_{H}^{2} \sigma^{2}+\bar{\psi}\left(i \gamma^{\mu} D_{\mu}-m_{f}\right) \psi+\mu_{f} \psi^{+} \psi
$$

Since the chemical potential is nonzero, $\tilde{A}_{0}$ has an expectation value, and this term plays the role of a tachyonic mass for the scalars (see, e.g., [9]). When $\left\langle 2 e \tilde{A}_{0}\right\rangle=m_{H}$, the scalar field condenses. We look at a solution with no boundary charge, for which $\left\langle A_{0}\right\rangle=0, \mu_{s}=m_{H}$ and

$$
\langle\sigma\rangle \equiv \sigma_{c}=\sqrt{\frac{J_{0}}{2 m_{H}}} .
$$

Hence, we're looking at the effects that are $1 / m_{H}$ suppressed.

\section{Static Potential Without Fermion Fluctuations}

The uniform background solution obtained above sets a preferred Lorentz frame. Let us look at small perturbations in this frame. We introduce perturbations of the gauge, $A_{\mu} \equiv b_{\mu}$, and the scalar field, as follows:

$$
\sigma(x)=\sigma_{c}+\tau(x)
$$

Let us for the time being ignore fermion fluctuations. Physically this would correspond to the case when fermions are "frozen in" (for instance if they form a rigid-enough crystalline structure). We will relax this conditions and include their fluctuation in the next Section.

Then, the Lagrangian density for the fluctuations in the quadratic approximation reads [1]

$$
\mathcal{L}_{2}=-\frac{1}{4} f_{\mu \nu}^{2}+\frac{1}{2}\left(\partial_{\mu} \tau\right)^{2}+\frac{1}{2} m_{\gamma}^{2} b_{\mu}^{2}+2 m_{H} m_{\gamma} b_{0} \tau
$$

Here

$$
m_{\gamma}^{2} \equiv(2 e)^{2} \frac{J_{0}}{2 m_{H}},
$$

$f_{\mu \nu}$ denotes the field strength for $b_{\mu}$, and we left out for simplicity all the fermionic terms. It is useful to integrate out the $\tau$ field from (16). The remaining Lagrangian takes the form:

$$
\mathcal{L}_{2}=-\frac{1}{4} f_{\mu \nu}^{2}+\frac{1}{2} m_{\gamma}^{2} b_{\mu}^{2}+\frac{1}{2} b_{0} \frac{\left(2 m_{H} m_{\gamma}\right)^{2}}{\square} b_{0}
$$

This Lagrangian contains four components of $b_{\mu}$, and no other fields. The first two terms in (18) are those of a usual massive photon with three degrees of freedom. The 
last term is unusual, as it gives rise to the dynamics to the timelike component of the gauge field. This term emerged due to the mixing of $b_{0}$ with the dynamical field $\tau$ in (16), and since we integrated out $\tau, b_{0}$ inherited its dynamics in a seemingly nonlocal way. The fact that there are no pathologies in (16) or (18), such as ghost and/or tachyons, can be seen by calculating the Hamiltonian density from (16):

$$
\mathcal{H}=\frac{\pi_{j}^{2}}{2}+\frac{f_{i j}^{2}}{4}+\frac{\left(\partial_{j} \pi_{j}-2 m_{H} m_{\gamma} \tau\right)^{2}}{2 m_{\gamma}^{2}}+\frac{P_{\tau}^{2}+\left(\partial_{j} \tau\right)^{2}}{2} .
$$

Here, $\pi_{j} \equiv-f_{0 j}$ and $P_{\tau} \equiv \partial_{0} \tau$, and the Hamiltonian is positive semi-definite.

The form of the Lagrangian (18) is most useful for calculating a propagator. Indeed, the inverse of the quadratic operator that appears in (18) has poles which describe all the four propagating degrees of freedom. This propagator, sandwiched between two conserved currents $J_{\mu}$ and $J_{\mu}^{\prime}$, takes the form:

$$
J_{0}\left(-p^{2}+m_{\gamma}^{2}+\left[1-\frac{\omega^{2}}{m_{\gamma}^{2}}\right] \frac{4 M^{4}}{-p^{2}}\right)^{-1} a J_{0}^{\prime}-J_{j}\left(-p^{2}+m_{\gamma}^{2}\right)^{-1} J_{j}^{\prime} .
$$

This describes two transverse photons with the mass $m_{\gamma}$, one heavy mode with mass $2 m_{H}$, and a light phonon (for their dispersion relations, without and with fermion fluctuations, see Refs. [1, 2] respectively). Here $a \equiv 1-4 M^{4} \omega^{2} / p^{2} m_{\gamma}^{2}\left(p^{2}-m_{\gamma}^{2}\right)$.

In particular, we are interested in a static potential, which takes the form

$$
G(\omega=0, \mathbf{k})=\left(\mathbf{k}^{2}+m_{\gamma}^{2}+\frac{4 M^{4}}{\mathbf{k}^{2}}\right)^{-1}
$$

where, we have used the notation $M \equiv \sqrt{m_{H} m_{\gamma}}$, and the first, second, and third terms on the r.h.s. of (21) are due to the respective terms in (18).

Interestingly, when $m_{H} \gg m_{\gamma}$, as is the case here, there is no scale at which the photon mass term in (21) would dominate: for $\mathbf{k}^{2} \gtrsim m_{\gamma}^{2}$ the mass term is sub-dominant to the $\mathbf{k}^{2}$ term, while for $\mathbf{k}^{2} \lesssim m_{\gamma}^{2}$ it is sub-dominant to the $M^{4} / \mathbf{k}^{2}$ term.

Let us rewrite the Green's function (21) in the following form:

$$
G(\mathbf{k}, \omega=0)=\left(\mathbf{k}^{2}+m_{\gamma}^{2}\right)^{-1}-\left(\mathbf{k}^{2}+m_{\gamma}^{2}+\frac{\mathbf{k}^{2}\left(\mathbf{k}^{2}+m_{\gamma}^{2}\right)^{2}}{4 M^{4}}\right)^{-1}
$$

The first term on the r.h.s. of (22) could be thought of as an instantaneous repulsive screened Coulomb (Yukawa) potential, while the second term as an attractive potential due to the phonon. The phonon here is somewhat peculiar - it mixes with the timelike component of the gauge field, and thus acquires an instantaneous piece, a part of which cancels the instantaneous gauge potential. This is reflected in (22) as exact cancellation of the residues of the pole at $\mathbf{k}^{2}+m_{0}^{2}=0$. The remaining instantaneous part is what's given in (21). 
As we have already mentioned in Section 1, the remaining two poles of the second term in (22), describe respectively the heavy state of mass $2 m_{H}$, which is unimportant for the low-energy dynamics, and a light state - the phonon [2]. Just to reiterate, for simplicity of discussions, in this work we're using a somewhat imprecise language and calling the whole second term in (22) the phonon contribution.

The key observation is that at scales larger than $1 / M$, which are of the primary interest, the phonon potential cancels the gauge potential with a high accuracy. Note that this cancellation is reliable at scales that are much greater than $J_{0}^{-1 / 3} \gg M^{-1}$, and takes place already at scales that are much shorter that the photon Compton wavelength $m_{\gamma}^{-1} \gg J_{0}^{-1 / 3}$ !

In a Lorentz-invariant theory having a negative sign in front of a propagator, such as the one in the second term in (22), would suggest the presence of a ghostlike state. However, this is not the case in a Lorentz-violating theory described by our Lagrangian (16) or (18). As we pointed out above, the Hamiltonian density of this theory is positive semi-definite, and hence, no ghost or tachyons are present. Moreover, consistently with ones expectation, the second term in (22) disappears in the limit $M \rightarrow 0$, where Lorentz invariance of (16) or (18) is restored.

The above described properties can also be seen by calculating the coordinate space potential (see, $[2])$ :

$$
V \equiv\left(Q_{1} e Q_{2} e\right) \int \frac{d^{3} \mathbf{k}}{(2 \pi)^{3}} e^{i \mathbf{k x}} G(\omega=0, \mathbf{k}) \propto \frac{Q_{1} Q_{2} \alpha_{\mathrm{em}} e^{-M r}}{r} \cos (M r)
$$

in which we assumed that $r=|\mathbf{x}| \gg 1 / M$ and $m_{\gamma} \ll M$. Such a potential was first derived in [2]. It is sign-indefinite and undergoes modulated oscillations between repulsion and attraction. There are an infinite number of points in the position space where the force between classical charges would vanish. These are points where

$$
\frac{d V}{d r}\left(r=r_{n}\right)=0, \quad n=1,2, \ldots
$$

The potential wells described by (24) are too shallow to introduce a significant quantum-dynamics for static impurities - any two charged probe particles (e.g., nuclear hydrogen or helium-3 impurities) separated by a distance $r_{n} \gtrsim J_{0}^{-1 / 3}$, where our calculations are reliable, would stay in a static equilibrium as long as $V\left(r_{n}\right)<0$. This is because their charges will be screened efficiently by the ambient condensate.

Before turning to the next section we make three important comments:

Comment 1: Concerns the decoupling of the helium-4 sates in the $m_{H} \rightarrow \infty$ limit. As was already mentioned in Sections 1 and 2, the decoupling takes place via "freezing" out of the phonon associated with the collective motion of the heavy modes. The fact that this indeed is the case can be seen by looking at the dispersion relation for the phonon which is obtained from the poles of the Green's function (21) in which nonzero $\omega$ is restored. In Refs. [1] and [2] the full dispersion relations for the 
phonon without and with fermion fluctuations was given respectively. The "freezing out" of the phonon takes place in both cases.

For simplicity we discuss here the case of [2]: For the relevant momenta $M^{2} \gg \mathbf{k}^{2}$ the dispersion relation reduces to $\omega \simeq m_{\gamma}\left(1+\mathbf{k}^{2}\left(\mathbf{k}^{2}-m_{\gamma}^{2}\right) / 8 M^{4}\right)$, and the phonon group velocity

$$
v_{\mathrm{gr}} \simeq \frac{m_{\gamma}|\mathbf{k}|\left(2 \mathbf{k}^{2}-m_{\gamma}^{2}\right)}{4 M^{4}}
$$

vanishes in the $m_{H} \rightarrow \infty$ limit.

Note that for $\mathbf{k}^{2} \simeq m_{\gamma}^{2} / 2$ the phonon group velocity vanishes for finite $m_{H}$. This describes a state of a nonzero momentum but zero group velocity. The energy of this state is also nonzero, and to a good approximation equals to $m_{\gamma}$. These properties are similar to those of a roton in superfluid helium II. Moreover, for excitations with $\mathbf{k}^{2}>m_{\gamma}^{2} / 2$ the group velocity is positive, while in the opposite case, $\mathbf{k}^{2}<m_{\gamma}^{2} / 2$, it becomes negative (i.e., the direction of the momentum and that of group velocity are opposite to each other). These excitations resemble the positive and negative group velocity rotons in superfluid helium II.

Comment 2: Concerns the issue of applicability of the linearized approximation (16) that we adopted in this work. For this we should restore back all the non-linear terms that were ignored in (16) and compare them with the linear ones retained there. Although this can be done in full generality, it is instructive to look at the dynamics of a longitudinal polarization of the massive vector field (i.e., the would-be Nambu-Goldstone boson) which determines the fastest growing non-linear terms in the Lagrangian at high energies $[16,17]$. This is easily achieved by employing the substitution $b_{\mu} \rightarrow\left(\partial_{\mu} n\right) / m_{\gamma}$, and taking the decoupling limit: $m_{\gamma} \rightarrow 0$ and $e \rightarrow 0$, while keeping the ratio $v \equiv m_{\gamma} / e$ finite and fixed. The resulting Lagrangian for the longitudinal mode $n$, and the remaining field $\tau$ takes the form

$$
\frac{\left(\partial_{\mu} n\right)^{2}}{2}+\frac{\left(\partial_{\mu} n\right)^{2} \tau}{v}+\frac{\left(\partial_{\mu} n\right)^{2} \tau^{2}}{2 v^{2}}+2 m_{H}\left(\partial_{0} n\right) \tau+\frac{m_{H}\left(\partial_{0} n\right) \tau^{2}}{v}+\frac{\left(\partial_{\mu} \tau\right)^{2}}{2} .
$$

From this we deduce that the non-linear terms become comparable with the linear ones when $\tau \sim v$; we also require that fluctuations of gauge field to be smaller tat the scalar chemical potential, which gives $\partial_{0} n \sim m_{H} v$. Thus, the domain of applicability of the linearized results of this work is

$$
\tau \ll v=\left(\frac{J_{0}}{2 m_{H}}\right)^{1 / 2}, \quad \partial_{0} n \ll m_{H} v=\left(J_{0} m_{H}\right)^{1 / 2} .
$$

In the limit $m_{H} \rightarrow \infty$, the domain of applicability of the linearized results shrinks to zero. This suggests that the geometric size of the region in which one can meaningfully talk about the charged condensate should be greater than a certain critical size that scales as $\sim v^{-1}$. The latter tends to infinity as $m_{H} \rightarrow \infty$. 
Comment 3: The dynamics of mall fluctuations can also be obtained from the effective Lagrangian (8). For this we restrict ourselves to the quadratic order in the expansion of $\mathcal{P}(x)$

$$
\mathcal{L}_{e f f}=2 e \Sigma^{2} B_{0}-\frac{\left(\nabla_{j} \Sigma\right)^{2}+(2 e)^{2} \Sigma^{2} B_{j}^{2}}{2 m_{H}}+C_{2}(2 e)^{2} \Sigma^{4} B_{0}^{2},
$$

and expand the above expression (amended with the gauge-kinetic and fermionic terms) to the second order in the following fluctuations:

$$
\Sigma=\sqrt{\frac{J_{0}}{2}}+\sqrt{m_{H}} \tau(x), \quad B_{\mu} \equiv b_{\mu}
$$

The resulting Lagrangian for the fluctuations reads:

$$
\mathcal{L}_{2}=-\frac{1}{4} f_{\mu \nu}^{2}-\frac{1}{2}\left(\partial_{j} \tau\right)^{2}+\frac{1}{2} m_{0}^{2} b_{0}^{2}-\frac{1}{2} m_{\gamma}^{2} b_{j}^{2}+2 m_{H} m_{\gamma} b_{0} \tau,
$$

where $m_{0}^{2}=2 e^{2} C_{2} J_{0}^{2}$ and, as before, $m_{\gamma}^{2}=(2 e)^{2} J_{0} / 2 m_{H}$. This should be compared with (16). We notice that unlike in (16), there is no kinetic term for $\tau$ in (30). This is because we decoupled the time-dependence of the heavy field in (6) in the nonrelativistic limit; thus, the $\Sigma$ field, or its fluctuation $\tau$, are left to be instantaneous. This, obviously, does not change the conclusions on the static potential discussed in this Section.

Furthermore, depending on the coefficient $C_{2}$ the "electric" and "magnetic" masses of the gauge field can be different. The value of $C_{2}$ cannot be fixed within the effective Lagrangian approach. One can only impose a bound on it. For instance, without the $b_{0}-\tau$ mixing, superluminal group velocity is avoided when ${ }^{7}$

$$
m_{0}^{2} \geq m_{\gamma}^{2}
$$

In this Section the value of $C_{2}$ was assumed to saturate the above inequality, as we put $m_{0}^{2}=m_{\gamma}^{2}$. However, the fermion fluctuations, which we ignored so far, will produce a large hierarchy between the coefficients of the $b_{0}^{2}$ and $b_{j}^{2}$ terms in the Lagrangian, even if these were assumed to be equal in the classical theory.

Finally, let us look at the decoupling limit: $e \rightarrow 0$, while $m_{0} / e$ and $m_{\gamma} / e$ are finite and fixed. Then, the Lagrangian (30) reduces to

$$
\frac{m_{0}^{2}}{2 m_{\gamma}^{2}}\left(\partial_{0} n\right)^{2}-\frac{1}{2}\left(\partial_{j} n\right)^{2}+2 m_{H}\left(\partial_{0} n\right) \tau-\frac{1}{2}\left(\partial_{j} \tau\right)^{2} .
$$

\footnotetext{
${ }^{7}$ Note that the longitudinal part of $b_{j}$ is a propagating mode. Imposing transversality on $b_{j}$ would contradict general equations of motion. Also, superluminal group velocity in the present case would suggest some instability of the background. In general, superluminal group velocities can exist in highly absorptive media, in which case group velocity does not approximate the velocity of propagation of a signal.
} 
This should be compared with the quadratic part of (32). Again, there is missing kinetic term for $\tau$, for reasons explained above. As before, $\tau$ can be integrated out. The resulting dispersion relation for the remaining mode reads

$$
\omega^{2} \simeq \frac{\mathbf{k}^{4}}{4 m_{H}^{2}+\mathbf{k}^{2}\left(m_{0}^{2} / m_{\gamma}^{2}\right)},
$$

which for small momenta, $\mathbf{k}^{2} \ll m_{H}^{2}\left(m_{\gamma}^{2} / m_{0}^{2}\right)$, gives $\omega \simeq \mathbf{k}^{2} / 2 m_{H}$, as it should be the case for a collection of free massive particles.

\section{Quantum Screening of Static Charges}

In the previous section the fermions were treated as "frozen". In may physical circumstances, and in particular in white dwarfs, this is not a good approximation. For the helium-electron system the fermion fluctuations should be taken into account. This was done in Ref. [2] using the Thomas-Fermi (TF) approximation. The result is that the timelike component of the gauge field acquired an additional mass - the electric mass. This breaks the degeneracy between the coefficients of $b_{0}^{2}$ and $b_{j}^{2}$ discussed in the previous Section.

However, the TF approximation does not capture one significant property of the fermion system related to a possibility of exciting gap-less modes near the Fermi surface, which we will incorporate below into our results by using the one-loop correction to the propagator of (18). For this, we restore back in the Lagrangian (18) the fermion kinetic, mass and chemical potential terms and, upon calculating the gauge boson propagator, will take into account the known one-loop gauge boson polarization diagram.

Since we're interested in a static potential, we look at the $\{00\}$ component of the propagator $D_{00}$, and the static potential obtained from it:

$$
\tilde{V}(k) \equiv-D_{00}(\omega=0, \mathbf{k})=\left(\mathbf{k}^{2}+m_{\gamma}^{2}+\frac{4 M^{4}}{\mathbf{k}^{2}}+F\left(k^{2}, k_{F}, m_{f}\right)\right)^{-1} .
$$

where the function $F\left(k^{2}, k_{F}, m_{f}\right)$, which is due to the one-loop photon polarization diagram, includes both the vacuum and fermion matter contributions $\left(k_{F}\right.$ denotes the Fermi momentum). A complete expression for $F\left(k^{2}, k_{F}, m_{f}\right)$ can be found in Ref. [5]. We concentrate on the expression for $F\left(k^{2}, k_{F}, m_{f}\right)$ in the massless $\left(m_{f}=0\right)$ limit that is a good approximation for ultra-relativistic fermions:

$$
F\left(\mathbf{k}^{2}, k_{F}\right)=\frac{e^{2}}{24 \pi^{2}}\left(16 k_{F}^{2}+\frac{k_{F}\left(4 k_{F}^{2}-3 k^{2}\right)}{k} \ln \left(\frac{2 k_{F}+\mathbf{k}}{2 k_{F}-\mathbf{k}}\right)^{2}-k^{2} \ln \left(\frac{k^{2}-4 k_{F}^{2}}{\mu_{0}^{2}}\right)^{2}\right) .
$$

Here $\mu_{0}$ stands for the normalization point that appears in the one-loop vacuum polarization diagram calculation. The function $F$ introduces a shift of the pole in the propagator, corresponding to the "electric mass" of the photon. This part of 
the pole can be incorporated via the TF approximation, as it was done in [2]. In addition, however, the function $F$ also gives rise to branch cuts in the complex $|\mathbf{k}|$ plane (see [3] for the list of earlier references on this).

As in the previous section we can decompose the static potential as follows:

$$
\tilde{V}(\mathbf{k}, \omega=0)=\left(\mathbf{k}^{2}+m_{\gamma}^{2}+F\right)^{-1}-\left(\mathbf{k}^{2}+m_{\gamma}^{2}+F+\frac{\mathbf{k}^{2}\left(\mathbf{k}^{2}+m_{\gamma}^{2}+F\right)^{2}}{4 M^{4}}\right)^{-1}
$$

Note that the first term in (36) is just the instantaneous screened-Coulomb (Yukawa) potential of a massive photon with the one-loop polarization correction. Our main interest is at distances smaller than $m_{\gamma}^{-1}$. A sphere of radius $m_{\gamma}^{-1}$ encloses many particles within its volume since $m_{\gamma}^{-1} \gg J_{0}^{-1 / 3}$. At these scales, the first term in (36) can be approximated by:

$$
\frac{1}{\mathbf{k}^{2}+F}
$$

The above expression has a regular pole corresponding to the acquired "electric" mass of the photon due to the polarization diagram. The contribution of this pole would give rise to an exponentially decaying potential $e^{-m_{\mathrm{el}} r} / r$, where $m_{\mathrm{el}} \sim e \mu_{f}$. This is just an ordinary Debye screening.

However, as was mentioned above, the expression (37) also has branch cuts in the complex $|\mathbf{k}|$ plane for $k= \pm 2 k_{F}$. These branch cuts give rise to the additional terms in the static potential which are not exponentially suppressed, but instead have an oscillatory behavior with a power-like decaying envelope. In a non-relativistic theory they're known as the Friedel oscillations [3]. In the relativistic theory they were calculated in Refs. [4, 5] (we follow here [5] and for simplicity ignore the running of the coupling constant due to the vacuum loop):

$$
\Delta V=\frac{Q_{1} Q_{2} \alpha_{\mathrm{em}}^{2}}{4 \pi} \frac{\sin \left(2 k_{F} r\right)}{k_{F}^{3} r^{4}}
$$

Note that these branch cuts have a physical interpretation: Since there is no mass gap in the fermion spectrum, a photon can produce a near-the-Fermi-surface particlehole pair of an arbitrarily small energy and the momentum close to $\pm 2 k_{F}$. The imaginary part of the one-loop photon polarization diagram should include the continuum of such near-the-Fermi-surface pairs. These are reflected as logarithmic branch cuts in the expression for $F$.

Thus, if the phonon term (the second term) on the r.h.s. of (36) were absent one would have a power-like behavior (38) of the static potential at scales $r \lesssim m_{\gamma}^{-1}$. The phonon term, however, significantly reduces the strength of this potential. The result for it could be calculated by directly taking Fourier transform of (34). The dominant contribution comes from the branch cuts at $k= \pm 2 k_{F}$. Drawing the contours around these cuts in the upper half plane of complex $|\mathbf{k}|[3,5]$, one deduces 
the result. In the approximation $M \gg k_{F} \gg m_{\gamma}$, which is relevant for our system, a static potential between like charges scales as

$$
\Delta V \simeq \frac{4 Q_{1} Q_{2} \alpha_{\mathrm{em}}^{2}}{\pi} \frac{k_{F}^{5} \sin \left(2 k_{F} r\right)}{M^{8} r^{4}}
$$

This potential has an additional suppression factor of $16\left(k_{F} / M\right)^{8} \sim 10^{-23}$, as compared to $\Delta V$ in a theory without phonons (38). Nevertheless, $\Delta V$ in (39) dominates over the exponentially suppressed part of the total potential found in Ref. [2] and discussed in the previous section (see, (23)), for separations between probe particles large-enough for the effective field theory description to be applicable. The net static interaction in the charged condensate, set by (39), is very weak. It is, however, still much stronger than gravitational interaction between a pair of light nuclei.

Although formally $\Delta V$ in (39) is proportional to $\alpha_{\mathrm{em}}^{2}$, to a good approximation it is independent of $\alpha_{\mathrm{em}}$ since $M^{8} \propto e^{4}\left(m_{H} J_{0}\right)^{2}$.

As in the previous section, the potential for the static charges (39) is shallow and sign-indefinite. There are, however, an infinite number of position-points where the force between static charges would vanish. These are determined by extremizing (39) and obtaining

$$
4 \tan \left(2 k_{F} r_{n}\right)=2 k_{F} r_{n}, \quad n=1,2,3, \ldots
$$

Some of the $r_{n}$ 's are local minima, and thus, static charges (e.g., helium-4 nuclei, nuclear hydrogen or helium-3 impurities) placed in those minima with $r_{n} \gtrsim J_{0}^{-1 / 3}$, where our approximations are valid, would stay in a local equilibrium due to efficient screening by the condensate. Hence, the condensate could tolerate some fraction of charged impurities before the latter could significantly affect the condensate itself.

Finite temperature corrections would modify these results quantitatively, however, the main point of efficient screening of the static charges in the condensate should remain valid at temperatures well-below the condensation point. For instance, in white dwarfs with temperature $10^{6}-10^{7} \mathrm{~K}$ we would expect the dominant temperature-dependent corrections to the potential to be proportional to $T / J_{0}^{1 / 3} \sim\left(10^{-4}-10^{-3}\right) \ll 1$, which are negligible.

\section{Acknowledgments}

GG is grateful to Juan Maldacena for stimulating questions, to Arkady Vainshtein for helpful discussions, and especially to Nima Arkani-Hamed for valuable conversations and comments. He also thanks A. Dolgov for sending the draft of [12] before its publication. The work of GG was partially supported by the NSF and NASA grants (PHY-0758032, NNGG05GH34G). RAR was supported by the James Arthur Graduate Assistantship at NYU. 


\section{References}

[1] G. Gabadadze and R. A. Rosen, Phys Lett. B 658 (2008), 266 [arXiv:0706.2304 [hep-th]].

[2] G. Gabadadze and R. A. Rosen, JCAP 0810, 030 (2008) [arXiv:0806.3692 [astro-ph]].

[3] A.L. Fetter, J.D. Walecka, "Quantum Theory of Many-Particle Systems", McGraw-Hill, 1971.

[4] H.D. Sivak, Physica 129A, 408 (1985).

[5] J. I. Kapusta and T. Toimela, Phys. Rev. D 37, 3731 (1988).

[6] M.R. Schafroth, Phys. Rev. 100, 463 (1955).

[7] L.L. Foldy, Phys. Rev. 124, 649 (1961).

[8] J.P. Hansen, B. Jancovici, D. Schiff, Phys. Rev. Lett. 29, 991 (1972).

[9] A. D. Linde, Phys. Rev. D 14, 3345 (1976).

[10] J. I. Kapusta, Phys. Rev. D 24 (1981) 426.

[11] H. E. Haber and H. A. Weldon, Phys. Rev. D 25, 502 (1982).

[12] A.D. Dolgov, A. Lepidi, G. Piccinelli, arXiv:0811.4406 (hep-th).

[13] J. Polchinski, arXiv:hep-th/9210046.

[14] E.M. Lifshitz, L.P. Pitaevskii, "Statistical Physics", part 2, (1980).

[15] M. Greiter, F. Wilczek and E. Witten, Mod. Phys. Lett. B 3, 903 (1989).

[16] A. I. Vainshtein and I. B. Khriplovich, Yad. Fiz. 13 (1971) 198.

[17] J. M. Cornwall, D. N. Levin and G. Tiktopoulos, Phys. Rev. D 10, 1145 (1974) [Erratum-ibid. D 11, 972 (1975)]. 